\title{
Energy management of a dual-motor driven city bus based on reformed dynamic programming
}

\author{
Wenwei Wang ${ }^{1}$, Hong $\operatorname{Pan}^{1}$, Lin Cheng ${ }^{1}$ \\ ${ }^{1}$ National Engineering Laboratory for Electric Vehicles, Beijing Institute of Technology, Beijing, 100081, China
}

\begin{abstract}
This paper proposes a reformed dynamic programming (DP) based energy management strategy for a city bus driven by dual-motor coupling propulsion system(DMCPS). An instantaneous optimal problem of DMCPS's total energy loss is constructed to solve the torque allocation between two motors. Taking the results as extra constraints, a reformed DP architecture aimed at optimal energy consumption is established, where the state variables are the battery's SOC and operating modes of DMCPS, with a sole decision variable of mode switching action. The optimization results show a close performance to the original method, with the calculation efficiency greatly improved and the calculation time reduced by nearly $97 \%$. To obtain practical rules for real-time application, the mode switching schedule is extracted based on a RBF-SVM classifier, and the torque allocation is ruled by linear function. Simulation results demonstrate that the extracted rules can be executed through an on-board processor, with energy consumption reduced by $2.19 \%$ compared to the original rule-based strategy.
\end{abstract}

\section{Introduction}

Dual-motor coupling propulsion system has great advantages in realizing the comprehensive performance of battery electric city bus, with great potential for energy saving [1]. And energy management strategy, which concludes torque allocation and mode switching, is one critical factor influencing its energy consumption. And due to its complex structure, a problem of balancing the strategy's optimum and practicability is proposed. Rule-based strategies have been widely utilized in practice with fast real-time calculation and good robust property [2-3]. However, it can hardly obtain the optimal energy consumption performance when it is only developed by empirical logic. On the contrary, optimization-based strategies based on dynamic programming can offer the global optimal policy, of which the real-time application is severely restricted by its large calculation workload [4-5]. Therefore, some researchers take offline global optimization results as reference to extract new control rules or recalibrate the original rules, which achieve a relative balance between both aspects [6-7].

In this paper, an energy management strategy for DMCPS based on reformed dynamic programming (DP) is proposed. In Section 2, the system configuration and operating modes are given and modeled. In Section 3, the reformed dynamic programming architecture is given, based on the instantaneous optimal torque allocation for the system's total energy loss. The rule extraction method based on the optimization results is also introduced to solve the energy management problem in a typical driving cycle. The simulation results are illustrated, compared and discussed in Section 4 before conclusions are drawn in the final section.

\section{Configuration, operating modes, and modelling of DMCPS}

The dual-motor coupling propulsion system (DMCPS) studied in this paper is a centralized drive system, as shown in Fig.1. Here an auxiliary motor (EM2) equipped with a two-speed planetary transmission (AMT) and a traction motor (EM1) connected directly to the drive axle can drive the vehicle in a torque-coupling way. It is noted that the EM2 can be turned off and completely isolated from the EM1. Three different operating modes are defined for the system and distinguished by the gears of AMT, which includes gear 1 and gear 2 of dual-motor mode and gear 0 for EM1-only mode. Efficiency models of AMT and motors are constructed based on experiments by look-up table. Rint model is utilized for the battery. Power balance is established through vehicle dynamics. 


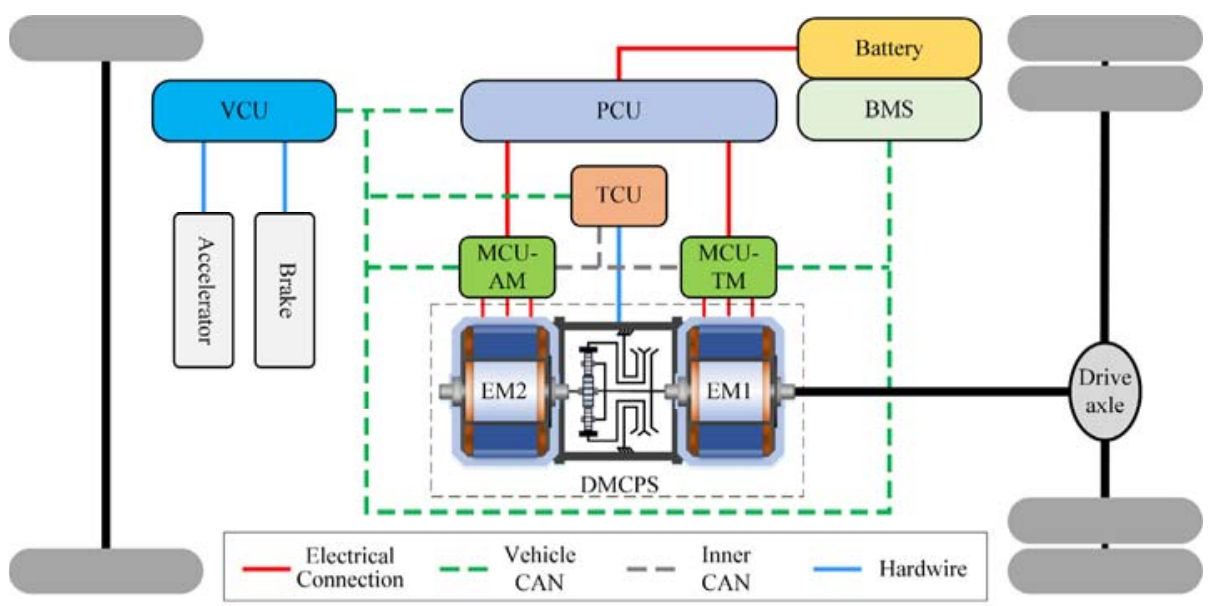

Fig.1 Configuration of the dual-motor coupling propulsion system

\section{Energy management based on reformed DP}

\subsection{The torque allocation based on instantaneous optimal problem}

$$
\left[T_{E M 1}^{*}(k), T_{E M 2}^{*}(k)\right]=\underset{\left.T_{E M}(k) \in T_{E M, \text {,in }}(k), T_{E M, \text { max }}(k)\right)}{\operatorname{argmin}}\left\{P_{E M 1, \text { lass }}(k)+P_{E M 2, \text { lass }}(k)+P_{A M T, \text { lass }}(k)\right\}
$$

where $T_{E M i}^{*}(k)$ is the optimal output torque and $P_{\text {EMiloss }}(k)$ is the power loss of each motor $\mathrm{Em} i, i=1,2$. $P_{A M T, \text { loss }}(k)$ is the power loss on transmission. In order to improve the calculation efficiency, the power loss of $\min f\left(T_{E M 1}(k), T_{E M 2}(k)\right)$

$$
\begin{aligned}
& =\left(\begin{array}{c}
T_{E M 1}(k) \\
T_{E M 2}(k)
\end{array}\right)^{T}\left(\begin{array}{cc}
a_{E M 1}(k) & 0 \\
0 & a_{E M 2}(k)
\end{array}\right)\left(\begin{array}{c}
T_{E M 1}(k) \\
T_{E M 2}(k)
\end{array}\right) \\
& +\left(\begin{array}{c}
b_{E M 1}(k) \\
b_{E M 2}(k)+\left(1-\eta_{g}(k)\right) \omega_{E M 2}(k)
\end{array}\right)^{T}\left(\begin{array}{c}
T_{E M 1}(k) \\
T_{E M 2}(k)
\end{array}\right)
\end{aligned}
$$

where, at moment $k, a_{E M i}(k), b_{E M i}(k)$ are the second-order and first-order coefficient of the power loss function of $\mathrm{Em} i, T_{\text {dem }}(k)$ is the total demand torque of the system, $i_{g}(k)$ is the ratio and $\eta_{g}(k)$ is the efficiency of the gear of AMT respectively.

\subsection{The reformed dynamic programming architecture}

The reformed DP based energy management strategy has the following similarities and differences compared with the original solution:

1) Considering key definitions, the original dynamic programming scheme contains 2 state variables (battery SOC, operating mode), 2 decision variables (mode switching command, torque allocation coefficient); The reformed scheme has the same 2 state variables, but only one decision variable which is the mode switching command. To avoid frequent mode switching, a penalty$$
\begin{aligned}
\text { subject to } & \left(\begin{array}{l}
T_{E M 1}(k) \\
T_{E M 2}(k)
\end{array}\right) \leq\left(\begin{array}{c}
T_{E M 1, \text { max }}(k) \\
T_{E M 2, \text { max }}(k)
\end{array}\right) \\
& \left(\begin{array}{c}
1 \\
i_{g}(k) \eta_{g}(k)
\end{array}\right)^{T}\left(\begin{array}{c}
T_{E M 1}(k) \\
T_{E M 2}(k)
\end{array}\right)=T_{d e m}(k)
\end{aligned}
$$

motor at a certain speed is fitted as a quadratic function of torque. Then the problem in Eq. (1) is equivalent to the following quadratic programming problem:

$$
\left(\begin{array}{c}
T_{E M 1}(k) \\
T_{E M 2}(k)
\end{array}\right) \geq\left(\begin{array}{l}
T_{E M 1, \min }(k) \\
T_{E M 2, \min }(k)
\end{array}\right)
$$

coefficient $\lambda$ is added in both scheme. So the expressions of reformed DP are as follows:

$$
\left\{\begin{array}{l}
x(k+1)=f(x(k), u(k)) \\
x(k)=[\operatorname{SOC}(k), \text { gear }(k)] \\
u(k)=\operatorname{shift}(k) \\
J=\sum_{0}^{N-1}\left[P_{\text {bat,total }}(x(k), u(k), k)+\lambda \operatorname{sgn}|\operatorname{shift}(k)|\right]
\end{array}\right.
$$

2) Considering the optimization process, for the original DP, both decision variables are optimized in a same step; Under the reformed framework, the operating mode is given at first, and then the optimal torque is determined by the instantaneous optimization during the backward solving process of DP, of which the flow chart is shown in Figure 2. 


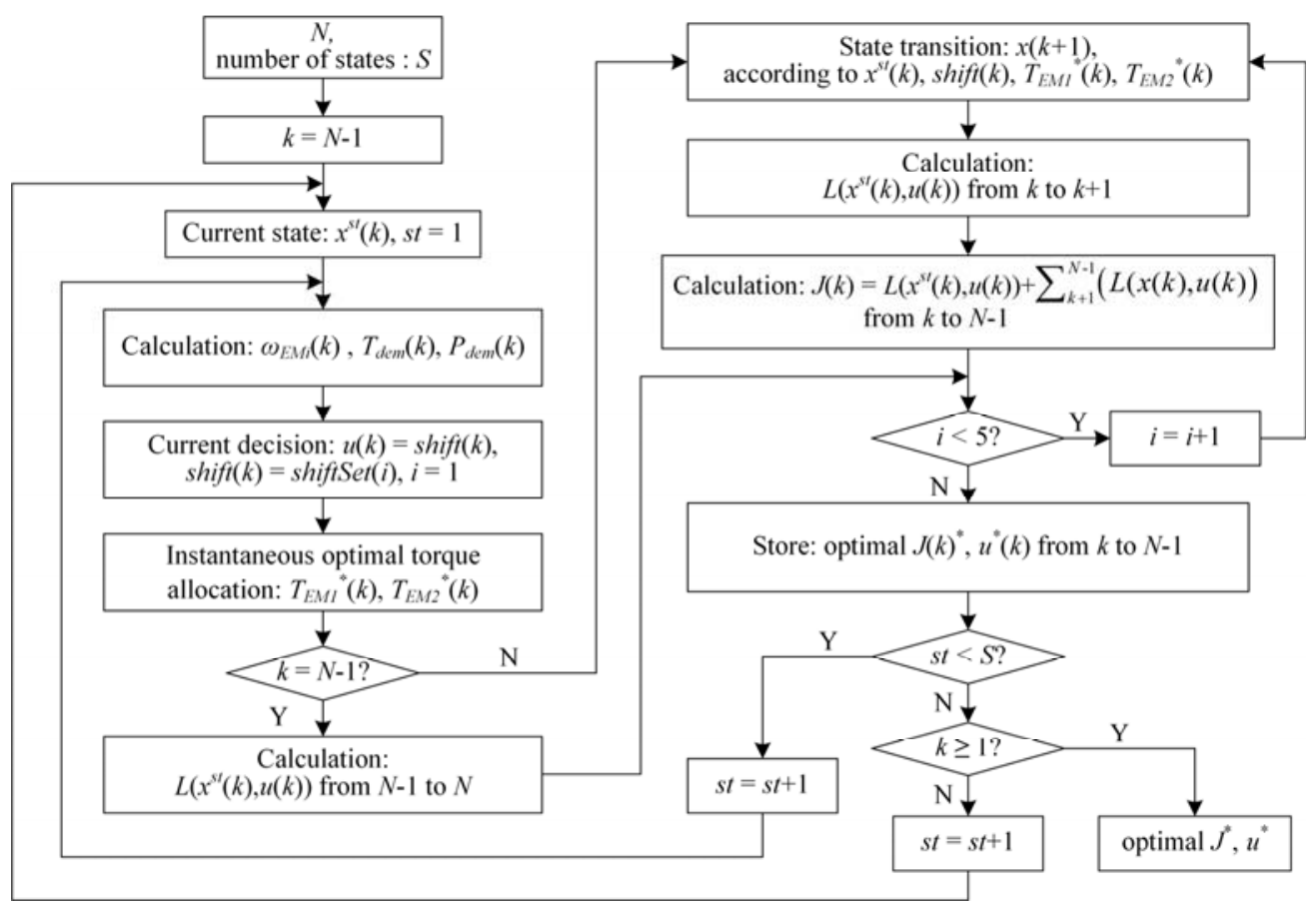

Fig.2 Backward solving process flow chart of reformed DP

3)The state and decision variables and $\lambda$ of reformed DP are discretized by minor certain grids and resolution, as shown in table 1 with basic properties.

Table.1 Key variables of reformed DP

\begin{tabular}{cccc}
\hline No. & Variables & Range & Resolution \\
\hline 1 & State of charge (SOC) & $0-1$ & 0.004 \\
2 & Gear of the transmission (gear) & $\{0,1,2\}$ & - \\
3 & Mode Switching command (shift) & $\{-2,-1,0,1,2\}$ & - \\
4 & Penalty coefficient $(\lambda)$ & $0-+\infty$ & 0.01 \\
\hline
\end{tabular}

\subsection{0ptimization results and rules extraction}

It is seen from the optimization results under C-WTVC shown in Fig. 3 that the reformed DP has a pretty close energy consumption performance with the original DP,

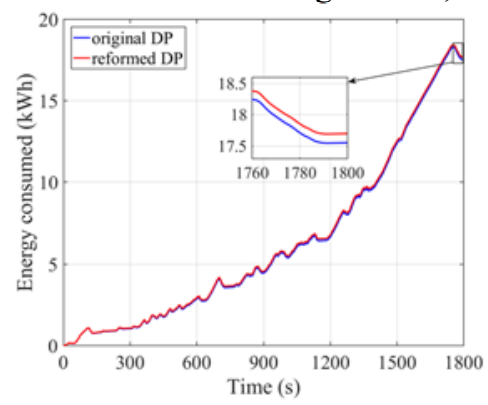

(a) Energy consumed which brings an increase of energy consumption by only $0.82 \%$. In an environment with a processor of $2.30 \mathrm{Ghz}$ and RAM of $8 \mathrm{~GB}$, the solution time of the reformed DP is $14.620 \mathrm{~s}$, reduced by $97 \%$ compared to $413.556 \mathrm{~s}$ of the original DP.

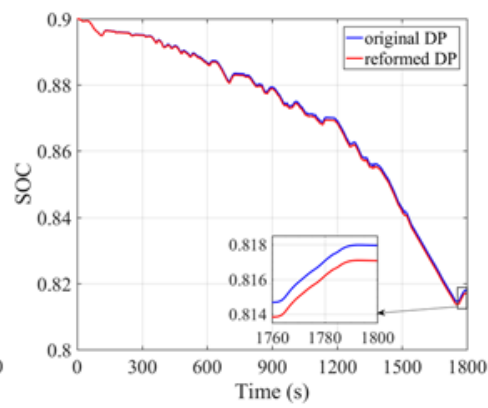

(b) $\mathrm{SOC}$

Fig. 3 Energy consumption results of DP with 2 different architecture 


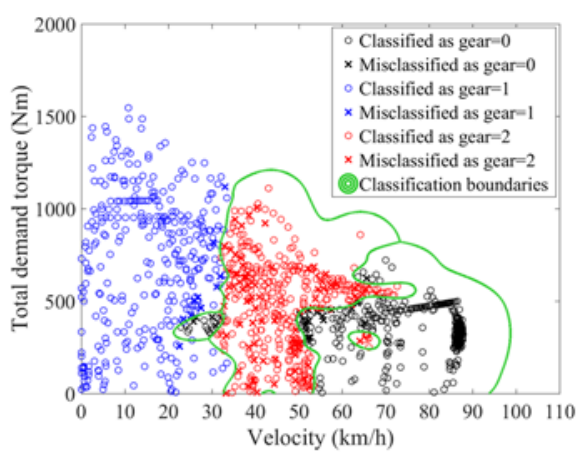

(a) Acceleration process

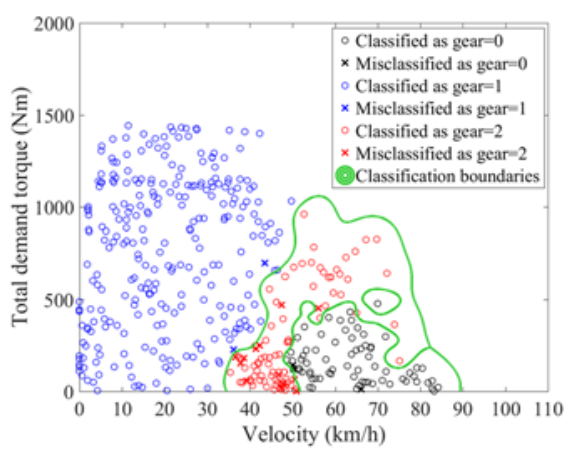

(b) Braking process

Fig.4 Classification results and boundaries of RBF-SVM classifier

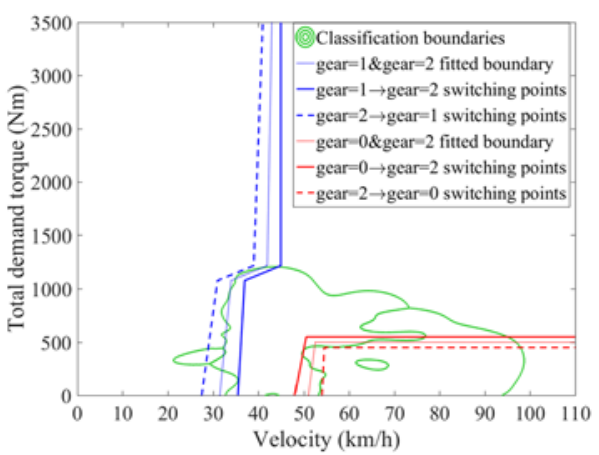

(a) Acceleration process

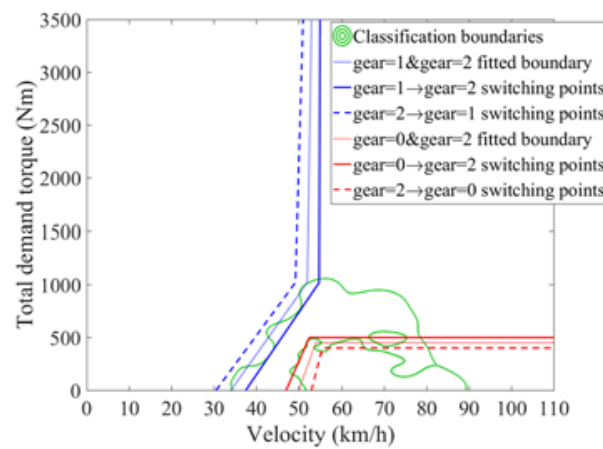

(b) Braking process

Fig.5 Mode switching rules based on classification boundaries

The mode switching rule is extracted based on a SVM classifier, of which the training set is the optimized operating modes distribution, the inputs are velocity and total demand torque, with a kernel function of radial basis function (RBF). The classification results and boundaries are shown in Fig.4. The results show that the accuracy of 10 -fold cross-validation in driving and braking process is $90.0 \%$ and $94.9 \%$ respectively. Then the fitted boundaries and switching points can be formulated based on the classification boundaries, as shown in Fig.5, considering necessary delays in practical application.

And for torque allocation rules, to improve the efficiency of real-time application, the linear function between the optimal torque of EM1 and the total demand torque at a series reference speeds obtained by offline optimization is stored and used in practice through a look-up table.

\section{Simulation results and comparison}

The above extracted energy management rules applied to an electric city bus are simulated under C-WTVC in a hardware-in-the-loop platform with a designed controller unit.

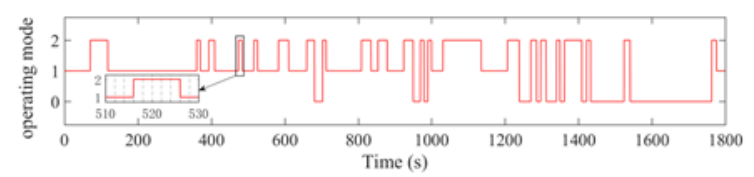

(a) Operating modes sequence

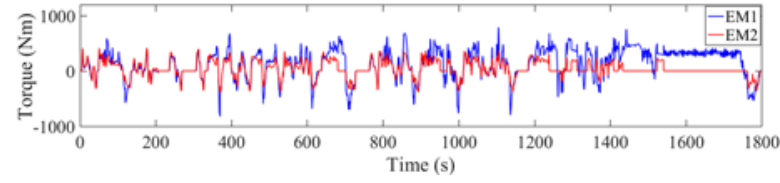

(b) Output torque of motors

Fig.6 Operating modes and motor torque results of extracted rules 


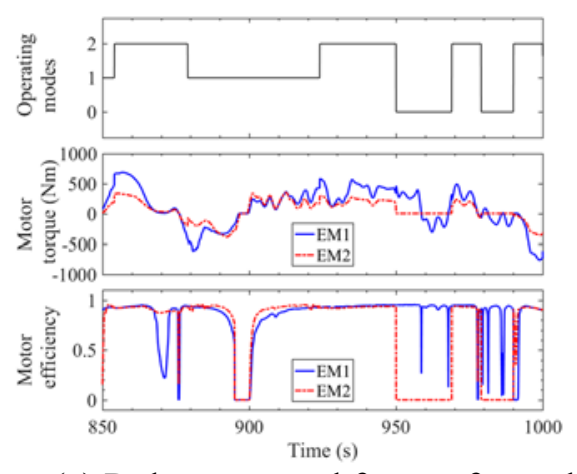

(a) Rules extracted from reformed DP

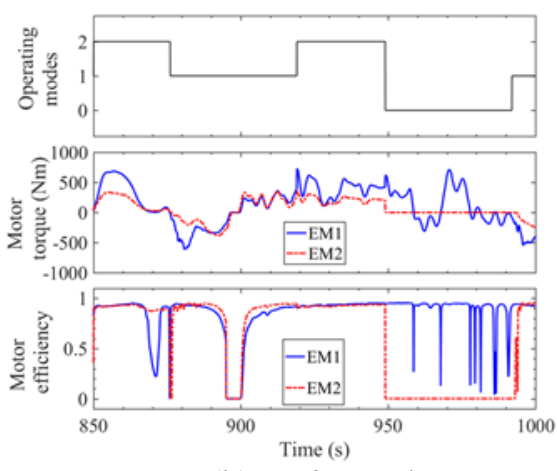

(b) Reformed DP

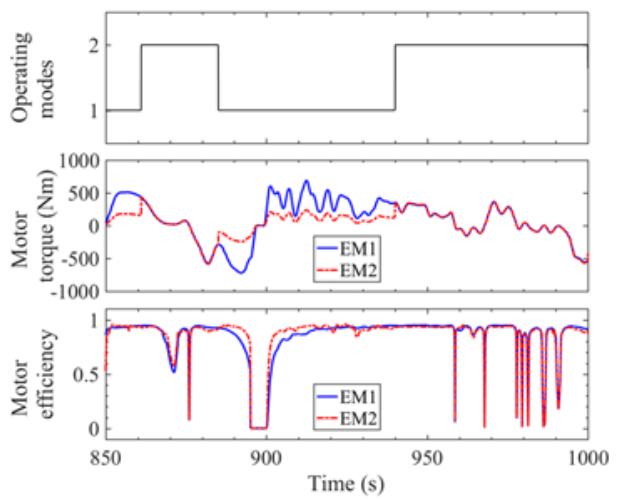

(c) Original rules

Fig.7 Results of a segment of the cycle of 3 different energy management strategies

It is seen from the results shown in Fig.6 that the operating mode under extracted rules has a reasonable switching frequency with a minimum interval of $10 \mathrm{~s}$, and the torque allocation results show a characteristic that the output of DMCPS is mainly provided by EM1 and supplemented by EM2. It is shown in Fig.7 that the extracted rules have a nearly same results, including the motors' efficiency, with the reformed DP, which indicates it can largely retain the control effect of the original strategy.

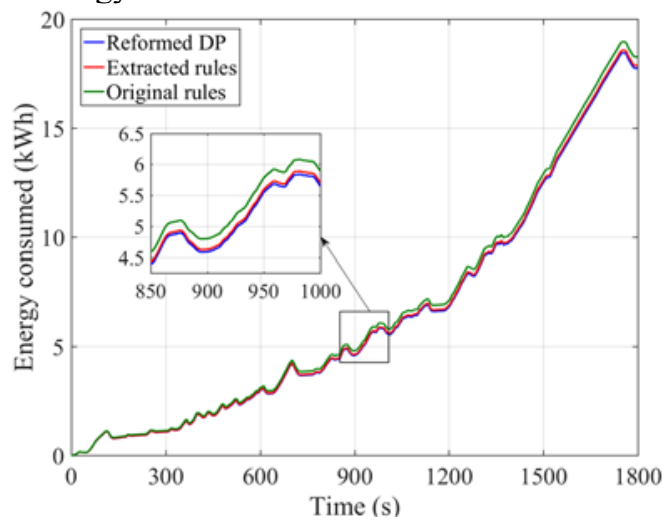

(a) Energy consumed
As is shown in Fig.8, the energy consumption per 100 kilometres of the extracted rules is $87.13 \mathrm{kWh}$, only $0.72 \%$ higher than the reformed DP, and $2.19 \%$ lower than the original empirical rules, achieving an energy saving of $1.95 \mathrm{kWh} / 100 \mathrm{~km}$; For the variation of SOC in a single cycle, the extraction rule is 0.0836 , reduced by 0.0019 compared to the original rules.

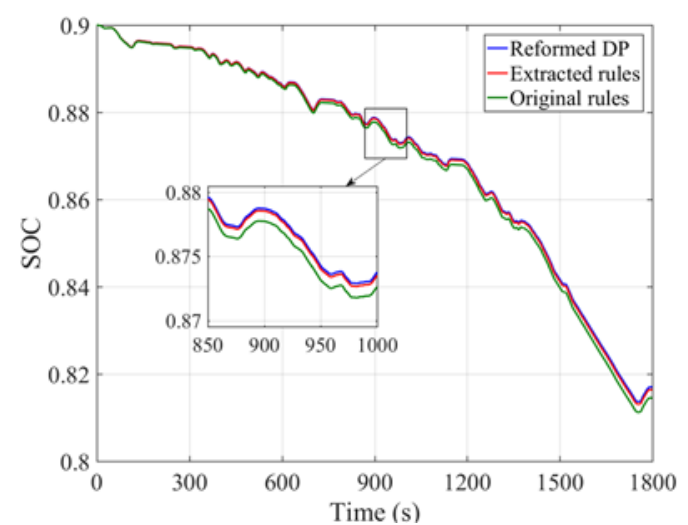

(b) SOC

Fig.8 Energy consumption performance of 3 different energy management strategies

\section{Conclusion}

Based on the results and discussions presented above, the conclusions are obtained as below:

(1) It is shown that, for energy management of DMCPS-driven bus, the reformed DP has a rather close optimization effect with normal DP, with energy

consumption increasing by $0.82 \%$, but achieves a significant reduction of calculation time of nearly $97 \%$.

(2) The rules extracted from reformed DP through the proposed method is proven to be suitable and effective for real-time application in a battery city bus, realizing an energy consumption reduction by $2.19 \%$ compared to the original rule-based strategy. 


\section{Acknowledgments}

This work was financially supported by the National Key Technology Research and Development Program of China (2017YFB0103801).

\section{References}

1. Zhao M, Shi J, et al. Application-oriented optimal shift schedule extraction for a dual-motor electric bus with automated manual transmission[J]. Energies, 2018, 11(2):325.

2. Wirasingha SG, Emadi A. Classification and review of control strategies for plug-in hybrid electric vehicles[J]. IEEE Transactions on Vehicular Technology, 2011, 60:111-22.

3. Padmarajan B V, Mcgordon A, et al. Blended rulebased energy management for phev: system structure and strategy[J]. IEEE Transactions on Vehicular Technology, 2016, 65(10):8757-8762.

4. Di Cairano S, Bernardini D, et al. Stochastic mpc with learning for driver-predictive vehicle control and its application to hev energy management[J]. IEEE Transactions on Control Systems Technology, 2014, 22(3):1018-1031.

5. Liu H, Han L, et al. Markov velocity predictor and radial basis function neural network based real-time energy management strategy for plug-in hybrid electric vehicles[J]. Energy, 2018, 152(Jun.1):427444.

6. Peng J, He H, et al. Rule based energy management strategy for a series-parallel plug-in hybrid electric bus optimized by dynamic programming[J]. Applied Energy, 2017, 185:1633-1643.

7. Shen W, Yu H, et al. Optimization of shift schedule for hybrid electric vehicle with automated manual transmission[J]. Energies, 2016, 9(3):220. 